\title{
A note on the Baker-Campbell-Hausdorff series in terms of right-nested commutators
}

\author{
Ana Arnal* $^{*} \quad$ Fernando Casas $^{\dagger} \quad$ Cristina Chiralt $^{\ddagger}$ \\ Institut de Matemàtiques i Aplicacions de Castelló (IMAC) and Departament de \\ Matemàtiques, Universitat Jaume I, E-12071 Castellón, Spain.
}

\begin{abstract}
We get compact expressions for the Baker-Campbell-Hausdorff series $Z=$ $\log \left(\mathrm{e}^{X} \mathrm{e}^{Y}\right)$ in terms of right-nested commutators. The reduction in the number of terms originates from two facts: (i) we use as a starting point an explicit expression directly involving independent commutators and (ii) we derive a complete set of identities arising among right-nested commutators. The procedure allows us to obtain the series with fewer terms than when expressed in the classical Hall basis at least up to terms of grade 10 .
\end{abstract}

Keywords: Baker-Campbell-Hausdorff formula, exponentials, commutators $\operatorname{MSC}(2010)$ : 22Exx, 17Bxx

\section{Introduction}

Exponentials of non-commuting operators appear in many areas of physics and mathematics, ranging from quantum mechanics to the theory of Lie groups and Lie algebras to the numerical analysis of differential equations. It is then natural to consider products of such exponentials and how to express such products as the exponential of a new operator. This of course is closely related with the celebrated Baker-CampbellHausdorff theorem [5].

In the most basic algebraic setting, one considers the associative algebra $\mathbb{K}\langle X, Y\rangle$ of formal power series in the non-commuting variables $X$ and $Y$ over a field $\mathbb{K}$ of

\footnotetext{
*Email: ana.arnal@uji.es. ORCD: 0000-0002-3283-3379

${ }^{\dagger}$ Email: Fernando.Casaseuji.es. ORCID: 0000-0002-6445-279X

${ }^{\ddagger}$ Email: chiralt@uji.es. ORCID: 0000-0003-0925-4034
} 
characteristic zero. Then $\mathrm{e}^{X} \mathrm{e}^{Y}=\mathrm{e}^{\Phi(X, Y)}$, with

$$
\begin{aligned}
\Phi(X, Y) & =\log \left(\mathrm{e}^{X} \mathrm{e}^{Y}\right)=\sum_{k \geq 1} \frac{(-1)^{k-1}}{k}\left(\mathrm{e}^{X} \mathrm{e}^{Y}-1\right)^{k}=\sum_{k \geq 1} \frac{(-1)^{k-1}}{k}\left(\sum_{p+q>0} \frac{X^{p} Y^{q}}{p ! q !}\right)^{k} \\
& =\sum_{k \geq 1} \frac{(-1)^{k-1}}{k} \sum \frac{X^{p_{1}} Y^{q_{1}} \ldots X^{p_{k}} Y^{q_{k}}}{p_{1} ! q_{1} ! \ldots p_{k} ! q_{k} !}
\end{aligned}
$$

where, in the last expression, the inner summation extends over all non-negative integers $p_{1}, q_{1}, \ldots, p_{k}, q_{k}$ for which $p_{i}+q_{i}>0(i=1,2, \ldots, k)$. The first terms read explicitly

$$
\begin{aligned}
& \Phi=\left(X+Y+X Y+\frac{1}{2} X^{2}+\frac{1}{2} Y^{2}+\cdots\right)-\frac{1}{2}\left(X Y+Y X+X^{2}+Y^{2}+\cdots\right)+\cdots \\
& =X+Y+\frac{1}{2}(X Y-Y X)+\cdots=X+Y+\frac{1}{2}[X, Y]+\cdots
\end{aligned}
$$

The Baker-Campbell-Hausdorff $(\mathrm{BCH})$ theorem states that $\Phi(X, Y)$ in (1) can be expressed as

$$
\Phi(X, Y)=X+Y+\sum_{m \geq 2} \Phi_{m}(X, Y)
$$

where $\Phi_{m}(X, Y)$ is a homogeneous Lie polynomial in $X$ and $Y$ of degree $m$, i.e., a linear combination of commutators of the form $\left[V_{1},\left[V_{2}, \ldots,\left[V_{m-1}, V_{m}\right] \ldots\right]\right]$ with $V_{i} \in\{X, Y\}$ for $1 \leq i \leq m$, the coefficients being rational constants. The formal power series (2) is called the Baker-Campbell-Hausdorff series, and plays a fundamental role not only in the theory of Lie groups and Lie algebras [5], but also in linear differential equations, control theory, quantum and statistical mechanics, and numerical analysis (see e.g. [4, 25, 26, 27, 28]).

An explicit expression for $\Phi_{n}$ in the BCH series was provided by Dynkin $[9,11]$ in the form

$$
\Phi_{m}(X, Y)=\sum_{p_{i}, q_{i}} \frac{(-1)^{m-1}}{m} \frac{\left[X^{p_{1}} Y^{q_{1}} \ldots X^{p_{m}} Y^{q_{m}}\right]}{\left(\sum_{i=1}^{m}\left(p_{i}+q_{i}\right)\right) p_{1} ! q_{1} ! \ldots p_{m} ! q_{m} !},
$$

where the summation is taken over all non-negative integers $p_{1}, q_{1}, \ldots, p_{m}, q_{m}$ such that $p_{1}+q_{1}>0, \ldots, p_{m}+q_{m}>0$ and $\left[X^{p_{1}} Y^{q_{1}} \ldots X^{p_{m}} Y^{q_{m}}\right]$ denotes the right-nested commutator based on the word $X^{p_{1}} Y^{q_{1}} \ldots X^{p_{m}} Y^{q_{m}}$. Thus, for instance,

$$
\left[X Y^{2} X^{2} Y\right] \equiv[X Y Y X X Y] \equiv[X,[Y,[Y,[X,[X, Y]]]]]
$$

Expression (3) can be used in principle to compute $\Phi_{n}$ in the BCH series up to any desired order. One should notice, however, that not all the terms are independent, due to the many existing redundancies. Thus, in particular, $\left[X^{3} Y^{1}\right]=\left[X^{1} Y^{0} X^{2} Y^{1}\right]=$ $[X,[X,[X, Y]]]$. An additional source of redundancies arises from the Jacobi identity [27]:

$$
\left[X_{1},\left[X_{2}, X_{3}\right]\right]+\left[X_{2},\left[X_{3}, X_{1}\right]\right]+\left[X_{3},\left[X_{1}, X_{2}\right]\right]=0
$$

for any three variables $X_{1}, X_{2}, X_{3}$, and other identities obtained from it. From this perspective, a procedure allowing to remove at once all the superfluous terms in (3) is of great value for practical applications. 
Although different procedures exist in the literature to construct the $\mathrm{BCH}$ series up to an arbitrary degree in terms of commutators, all of them have a basic limitation, as is the case with the Dynkin presentation (3): not all the commutators are independent, and so a rewriting process has to be carried out to express the results in terms of a basis of the free Lie algebra $\mathcal{L}(X, Y)$ generated by $X$ and $Y$. This process, of course, although can be carried out by computer algebra systems, requires a good deal of computational time and memory resources. One of the most efficient algorithms was proposed in [7], where explicit expressions of $\Phi_{m}$ up to $m=20$ in terms of the classical Hall and Lyndon bases of $\mathcal{L}(X, Y)$ were obtained with relatively modest computer requirements. In any event, the fact that no specific bases in the free Lie algebra have been constructed in which the calculation of the BCH series is simplified constitutes a major difficulty when dealing with problems where this series plays a role [6].

Expressing the $\mathrm{BCH}$ series in terms of right-nested commutators presents several advantages, especially when the series is considered in some particular physical settings. There are problems whose structure leads in a natural way to consider the $\mathrm{BCH}$ series of two operators $X, Y$ satisfying $[Y,[Y,[X, Y]]] \equiv 0$. This happens, in particular, when designing splitting methods for the numerical integration of classical Hamiltonian systems and also for the time-dependent Schrödinger equation. Although in this case it is still possible to construct a generalized Hall basis [20], it is much simpler to identify the non-vanishing terms when using right-nested commutators. On the other hand, and contrary to Hall-Viennot bases, there is not a straightforward procedure to construct a set of independent right-nested commutators generating each homogeneous subspace of $\mathcal{L}(X, Y)$.

Several attempts have been made to directly remove in (3) redundant terms and therefore to express $\Phi_{m}$ only as a linear combination of independent right-nested commutators. Thus, we can mention in particular references [22] and [16], where compact expressions up to $m=8$ and $m=9$, respectively, have been reported, after identifying highly non-trivial commutators identities arising when $m \geq 4$.

In this work we show that it is indeed possible to get directly rather compact expressions for $\Phi_{m}$ in terms of right-nested commutators without much computational effort, sometimes with fewer terms than when expressed in the classical Hall basis. This reduction is still more remarkable if the existing commutator identities are introduced at any degree. In addition, the procedure can be easily extended to the $\mathrm{BCH}$ series involving any number of variables,

$$
\exp \left(X_{1}\right) \exp \left(X_{2}\right) \cdots \exp \left(X_{n}\right)=\exp \left(\Phi\left(X_{1}, X_{2}, \ldots, X_{n}\right)\right)
$$

This can be achieved by considering, instead of the Dynkin presentation (3), another explicit expression of $\Phi_{m}$ as a linear combination of products of $m$ operators $X$ and $Y$ ordered according with the group of permutations. It turns out that such a formula was also originally obtained by Dynkin and published in his somehow unnoticed paper [10] (see also the lecture notes by M. Müger [21] for a translation and a detailed derivation of all the results contained in the paper).

One could also consider, of course, left-nested commutators instead and the same results would be still valid with a factor $(-1)^{k}$, if $k$ is the number of nested commutators. 


\section{The $\mathrm{BCH}$ series in terms of permutations}

We consider the general case (5), i.e.,

$$
\Phi\left(X_{1}, X_{2}, \ldots, X_{n}\right)=\sum_{m \geq 1} \Phi_{m}\left(X_{1}, \ldots, X_{n}\right),
$$

where $\Phi_{m}\left(X_{1}, \ldots, X_{n}\right)$ is a homogeneous polynomial of degree $m$ in the non-commutative variables $X_{1}, \ldots, X_{n}$.

Let us denote by $\varphi_{n}\left(X_{1}, X_{2}, \ldots, X_{n}\right)$ the multilinear part of $\Phi_{n}\left(X_{1}, \ldots, X_{n}\right)$, i.e., the part obtained by replacing $X_{i}^{2}$ by 0 for all $i$ in $\Phi_{n}\left(X_{1}, \ldots, X_{n}\right)$. Then one has the following remarkable result (see e.g. [19]):

Proposition 1 It holds that

$$
\Phi_{m}\left(X_{1}, \ldots, X_{n}\right)=\sum_{\substack{i_{1}+\cdots+i_{n}=m \\ i_{j} \geq 0}} \frac{1}{i_{1} ! \cdots i_{n} !} \varphi_{m}(\underbrace{X_{1}, \ldots, X_{1}}_{i_{1}}, \ldots, \underbrace{X_{n}, \ldots, X_{n}}_{i_{n}})
$$

As far as we know, the first proof of Proposition 1 is due to Dynkin [10]. Later on, it was shown that the notion of Eulerian idempotent leads to a shorter proof $[19,6]$. The explicit expression of $\varphi_{n}\left(X_{1}, X_{2}, \ldots, X_{n}\right)$ can be obtained as follows [19]. Since we are only interested in the multilinear part of $\Phi_{n}$, we have to replace

$$
\exp \left(X_{1}\right) \exp \left(X_{2}\right) \cdots \exp \left(X_{n}\right)
$$

by

$$
\left(1+X_{1}\right)\left(1+X_{2}\right) \cdots\left(1+X_{n}\right)
$$

and analyze $\log \left(\left(1+X_{1}\right)\left(1+X_{2}\right) \cdots\left(1+X_{n}\right)\right)$, or more specifically, its multilinear part. In other words, we have to deal with $\log (1+Z)$, where

$$
Z=\sum_{i} X_{i}+\sum_{i<j} X_{i} X_{j}+\sum_{i<j<k} X_{i} X_{j} X_{k}+\cdots+X_{1} X_{2} \cdots X_{n}
$$

It is then clear that $\varphi_{n}$ is of the form

$$
\varphi_{n}\left(X_{1}, X_{2}, \ldots, X_{n}\right)=\sum_{\sigma \in S_{n}} c_{\sigma} X_{\sigma(1)} X_{\sigma(2)} \cdots X_{\sigma(n)}
$$

where the sum is extended over all permutations $\sigma$ of $\{1,2, \ldots, n\}$. The coefficients $c_{\sigma}$ in (8) can be obtained by analyzing the contribution coming from each power $Z^{k}$ in the expansion $\log (1+Z)=Z-\frac{Z^{2}}{2}+\cdots+\frac{(-1)^{k-1}}{k} Z^{k}+\cdots$. The situation is similar to the computation of the explicit expression for the Magnus expansion as carried out, in particular, in [26]: it turns out that

$$
c_{\sigma}=\sum_{\substack{i_{1}+\ldots+i_{m}=n \\ \sigma \in S\left(i_{1}, \ldots, i_{m}\right)}} \frac{(-1)^{m-1}}{m}
$$

where the sum is extended over all ordered partitions $i_{1}+\cdots+i_{m}=n$ of $n$ such that $\sigma \in S\left(i_{1}, \ldots, i_{m}\right)$, with

$S\left(i_{1}, \ldots, i_{m}\right)=\left\{\sigma \in S_{n} \mid \sigma(j)<\sigma(j+1)\right.$ for all $\left.j \neq i_{1}+\cdots+i_{\ell}, \quad \ell=1, \ldots, m-1\right\}$ 
and $S_{n}$ denotes the permutation group. A straightforward computation shows that this number is $\left(\begin{array}{c}n-d_{\sigma}-1 \\ m-1-d_{\sigma}\end{array}\right)$, where $d_{\sigma}$ is the number of descents in $\sigma$. We recall that $\sigma \in S_{n}$ has an ascent in $i$ if $\sigma(i)<\sigma(i+1), i=1, \ldots, n-1$ and it has a descent in $i$ if $\sigma(i)>\sigma(i+1)$.

In this way one arrives at $[26,19]$

$$
c_{\sigma}=\sum_{m=d_{\sigma}+1}^{n} \frac{(-1)^{m-1}}{m}\left(\begin{array}{c}
n-d_{\sigma}-1 \\
m-1-d_{\sigma}
\end{array}\right)=\frac{(-1)^{d_{\sigma}}}{n} \frac{1}{\left(\begin{array}{c}
n-1 \\
d_{\sigma}
\end{array}\right)}
$$

and finally

$$
\varphi_{n}\left(X_{1}, X_{2}, \ldots X_{n}\right)=\frac{1}{n} \sum_{\sigma \in S_{n}}(-1)^{d_{\sigma}} \frac{1}{\left(\begin{array}{c}
n-1 \\
d_{\sigma}
\end{array}\right)} X_{\sigma(1)} X_{\sigma(2)} \cdots X_{\sigma(n)} .
$$

At this point some remarks are in order:

- The existing relationship between the multilinear part $\varphi_{n}$ with the Eulerian idempotent can be both ways: either to compute the coefficients $c_{\sigma}$ in (8) by applying different descriptions of this object $[24,18]$ or by providing an explicit combinatorial expression for this Eulerian idempotent with (9) that allows in particular to characterize its symmetries [6].

- Goldberg [12] analyzed the formal power series (5) when $n=2$ characterizing the coefficient of the general term $X_{1}^{s_{1}} X_{2}^{s_{2}} \cdots$ in terms of certain polynomials. This result was generalized to an arbitrary $n$ in [15] (see also [14]). It turns out that the coefficients $c_{\sigma}$ and the explicit expression (9) reproduce these previous results.

If we restrict ourselves to the case of two variables $X_{1} \equiv X, X_{2} \equiv Y$, then eq. (7) reads

$$
\Phi_{m}(X, Y)=\sum_{\substack{i+j=m \\ i, j \geq 1}} \frac{1}{i !} \frac{1}{j !} \varphi_{m}(\underbrace{X, \ldots, X}_{i}, \underbrace{Y, \ldots, Y}_{j}),
$$

and, according with eq. (9), one gets for the first terms

$$
\begin{aligned}
\Phi_{2}(X, Y) & =\varphi_{2}(X, Y)=\frac{1}{2} X Y-\frac{1}{2} Y X \\
\Phi_{3}(X, Y) & =\frac{1}{2} \varphi_{3}(X, X, Y)+\frac{1}{2} \varphi_{3}(X, Y, Y) \\
& =\frac{1}{12} X X Y-\frac{1}{6} X Y X+\frac{1}{12} X Y Y+\frac{1}{12} Y X X-\frac{1}{6} Y X Y+\frac{1}{12} Y Y X \\
\Phi_{4}(X, Y) & =\frac{1}{6} \varphi_{4}(X, X, X, Y)+\frac{1}{4} \varphi_{4}(X, X, Y, Y)+\frac{1}{6} \varphi_{4}(X, Y, Y, Y) \\
& =\frac{1}{24} X X Y Y-\frac{1}{12} X Y X Y+\frac{1}{12} Y X Y X-\frac{1}{24} Y Y X X
\end{aligned}
$$




\section{The BCH series in terms of commutators}

Application of Dynkin-Specht-Wever (DSW) theorem to (9) allows one to express $\varphi_{n}\left(X_{1}, \ldots, X_{n}\right)$ in terms of commutators and get an alternative expression for the homogeneous Lie polynomial $\Phi_{n}$. If we define the Lie bracketing from right to left by the unique linear map $r$ such that for any word $w=a_{1} a_{2} \ldots a_{n-1} a_{n}$ of length $n$ one has $r(w)=\left[a_{1},\left[a_{2}, \ldots,\left[a_{n-1}, a_{n}\right] \cdots\right]\right]$, the DSW theorem states that for each homogeneous Lie polynomial $P$ of degree $n$, it is true that $r(P)=n P$ [23]. In consequence,

$\varphi_{n}\left(X_{1}, X_{2}, \ldots, X_{n}\right)=\frac{1}{n^{2}} \sum_{\sigma \in S_{n}}(-1)^{d_{\sigma}} \frac{1}{\left(\begin{array}{c}n-1 \\ d_{\sigma}\end{array}\right)}\left[X_{\sigma(1)},\left[X_{\sigma(2)} \cdots\left[X_{\sigma(n-1)}, X_{\sigma(n)}\right] \cdots\right]\right]$.

Notice that, as is the case with (3), not all the commutators (Lie brackets) appearing in (12) are linearly independent, due to antisimmetry and the Jacobi identity. Thus, if one aims to get an expression in terms of independent commutators, then a particular basis of the vector subspace spanned by those commutators in which each generator appears exactly once has to be considered. If we denote this subspace by $L_{n}\left(X_{1}, \ldots, X_{n}\right)$, then $\operatorname{dim} L_{n}=(n-1)$ !.

Among the possible bases of $L_{n}$, the class considered by Dragt \& Forest [8] is particularly appropriate. In forming such a basis, one uses only those right-nested brackets ending with a particular but otherwise arbitrary variable selected from the collection $X_{1}, X_{2}, \ldots, X_{n}$. If this variable is chosen as $X_{n}$, then the basis is formed by the right-nested brackets of the form

$$
\left[X_{k},\left[X_{j}, \ldots\left[X_{i}, X_{n}\right] \ldots\right]\right]
$$

where the indices $k, j, \ldots i$ are all possible permutations of $\{1,2, \ldots n-1\}$. Of course, there are $n$ different such bases, depending on the particular ending operator one selects. What makes this class of bases specially compelling is the following property. Suppose we have an expression in terms of products of $n$ distinct operators $X_{1}, \ldots, X_{n}$ which is known to be written as a linear combination of right-nested commutators in $L_{n}\left(X_{1}, \ldots, X_{n}\right)$. This is the case, in particular, of $\varphi_{n}$. Suppose all the right-nested commutators ending with, say, $X_{n}$ are used as a basis. Then, in this linear combination, the coefficient of the right-nested commutator $\left[X_{k},\left[X_{j}, \ldots\left[X_{i}, X_{n}\right] \ldots\right]\right]$ is precisely the coefficient of the permutation $\alpha=(k j \ldots i n)$ in the original expression.

In consequence, if we apply this observation to eq. (9), we end up with

$\varphi_{n}\left(X_{1}, X_{2}, \ldots, X_{n}\right)=\frac{1}{n} \sum_{\sigma \in S_{n-1}}(-1)^{d_{\sigma}} \frac{1}{\left(\begin{array}{c}n-1 \\ d_{\sigma}\end{array}\right)}\left[X_{\sigma(1)},\left[X_{\sigma(2)}, \cdots,\left[X_{\sigma(n-1)}, X_{n}\right] \cdots\right]\right]$,

just involving the $(n-1)$ ! permutations of $S_{n-1}$. Of course, similar formulas can be obtained for $\varphi_{n}$ if instead of choosing $X_{n}$ as the last element in the right-nested commutator of the basis one takes any other element $X_{j}, j=1, \ldots, n-1$. In any case, a more compact expression than (12) for $\varphi_{n}$ is obtained in this way, since all the commutators are now independent. 


\begin{tabular}{|c|ccccccccc|}
\hline$m$ & 2 & 3 & 4 & 5 & 6 & 7 & 8 & 9 & 10 \\
\hline \hline $\operatorname{dim} \mathcal{L}_{m}(X, Y)$ & 1 & 2 & 3 & 6 & 9 & 18 & 30 & 56 & 99 \\
\hline \# terms Hall basis & 1 & 2 & 1 & 6 & 6 & 18 & 24 & 56 & 86 \\
\# terms Lyndon basis & 1 & 2 & 1 & 6 & 5 & 18 & 17 & 55 & 55 \\
\hline no identities & 1 & 2 & 1 & 8 & 7 & 32 & 31 & 96 & 97 \\
Grade 4 & 1 & 2 & 1 & 6 & 5 & 24 & 23 & 78 & 78 \\
Grade 6 & 1 & 2 & 1 & 6 & 4 & 18 & 17 & 67 & 65 \\
Compact & 1 & 2 & 1 & 6 & 4 & 18 & 13 & 38 & 52 \\
\hline Symmetric compact & 0 & 2 & 0 & 6 & 0 & 18 & 0 & 42 & 0 \\
\hline
\end{tabular}

Table 1: Number of terms in the homogeneous Lie polynomial $\Phi_{m}(X, Y)$ in the BCH series for the first values of $m$, together with the dimension of $\mathcal{L}_{m}(X, Y)$. The last line refers to the symmetric BCH series, eq. (16).

\section{Reducing the number of commutators}

Applying formula (13) and taking into account eq. (10) to the case of two variables we get

$$
\Phi_{m}(X, Y)=\sum_{\substack{i+j=m \\
i, j \geq 1}} \frac{1}{i !} \frac{1}{j !} \frac{1}{m} \sum_{\sigma \in S_{m-1}} \frac{(-1)^{d_{\sigma}}}{\left(\begin{array}{c}
m-1 \\
d_{\sigma}
\end{array}\right)}\left[X_{\sigma(1)},\left[X_{\sigma(2)}, \cdots,\left[X_{\sigma(m-1)}, Y\right] \cdots\right]\right],
$$

where $X_{\sigma(i)}, i=1, \ldots, m-1$, can be either $X$ or $Y$ according with the particular permutation $\sigma$ considered. In particular, for the first terms we have

$$
\begin{aligned}
\Phi_{2}(X, Y)= & \frac{1}{2}[X, Y] \\
\Phi_{3}(X, Y)= & \frac{1}{12}[X,[X, Y]]+\frac{1}{6}[X,[Y, Y]]-\frac{1}{12}[Y,[X, Y]] \\
\Phi_{4}(X, Y)= & \frac{1}{24}[X,[X,[Y, Y]]]-\frac{1}{24}[X,[Y,[X, Y]]]+\frac{1}{36}[X,[Y,[Y, Y]]]- \\
& -\frac{1}{36}[Y,[X,[Y, Y]]]
\end{aligned}
$$

If we take into account, however, the obvious property $[z, z]=0$, then we get the correct formula for $\Phi_{m}(x, y)$ up to $m=4$ and a much reduced number of terms in $\Phi_{m}$ for $m \geq 5$ than formula (2) with (3). To substantiate this claim, we have elaborated the code presented in Appendix A for the computation of $\varphi_{m}$ and the functions $\Phi_{m}(X, Y)$. The number of terms in $\Phi_{m}$ produced by this code is collected in the fifth line of Table 1 up to $m=10$. It is labelled as "no identities" to emphasize the fact that no existing identities among commutators have been yet implemented. We include for comparison the corresponding number of terms of $\Phi_{m}(X, Y)$ in the classical Hall (third line) and Lyndon bases (fourth line) as obtained by applying the procedure of [7]. For completeness, we also write the dimension of each homogeneous subspace $\mathcal{L}_{m}(X, Y)$ of the free Lie algebra $\mathcal{L}(X, Y)$.

If we incorporate the identity

$$
[Y,[X,[X, Y]]]=[X,[Y,[X, Y]]]
$$


appearing at $m=4$ into the procedure, we get the numbers collected in the line labelled "Grade 4". It is remarkable that this number agrees with the one corresponding to the Lyndon basis up to $m=6$, whereas $\Phi_{8}$ and even $\Phi_{10}$ contain a smaller number of terms than in the classical Hall basis.

Whereas no further identities exist at $m=5$, the following three appear at $m=6$, namely [22]

$$
\begin{aligned}
& {[X, X, X, Y, X, Y]-2[X, Y, X, X, X, Y]+[Y, X, X, X, X, Y]=0,} \\
& {[X, X, Y, Y, X, Y]+3[Y, X, X, Y, X, Y]-3[X, Y, X, Y, X, Y]} \\
& \quad-[Y, Y, X, X, X, Y]=0, \\
& {[Y, Y, X, Y, X, Y]-2[Y, X, Y, Y, X, Y]+[X, Y, Y, Y, X, Y]=0,}
\end{aligned}
$$

where we have denoted $[X, X, X, Y, X, Y]:=[X,[X,[X,[Y,[X, Y]]]]]$, etc., for simplicity. By incorporating them into the algorithm we get a further reduction, as the line labelled "Grade 6" in Table 1 clearly shows.

As a matter of fact, a systematic procedure to generate all the existing identities at a given $m$ can be designed by using tools of linear algebra, and in particular Gaussian elimination, as is explained in Appendix B (see also [17]). The algorithm can also be used to construct bases of the homogeneous subspace $\mathcal{L}_{m}(X, Y)$ for any $m \geq 1$ formed by right-nested commutators in a quite straightforward manner. For completeness, we have collected all such existing identities up to $m=10$ in the reference [1].

Once the identities have been obtained, to get compact expressions a particular basis has to be identified at each $m$ so that the number of vanishing coefficients of $\Phi_{m}(X, Y)$ is as large as possible. This can be done either by inspection (for small $m$ ) or applying the technique proposed in [16]. By proceeding in this way, we have been able to get rather compact expressions for $\Phi_{m}$, as shown in Table 1 (line labelled "Compact"). The corresponding explicit expressions can be found in [1]. In the reduction process a relevant role is played by the existing symmetries, namely

$$
\begin{aligned}
& \Phi_{m}(-X,-Y)=(-1)^{m} \Phi_{m}(X, Y) \\
& \Phi_{m}(X, Y)=(-1)^{m+1} \Phi_{m}(Y, X)
\end{aligned}
$$

\section{Further considerations}

The above procedure can also be easily generalized to any number of variables. In particular, from eqs. (7) and (13) we get for the case of three variables

$$
\begin{aligned}
& \Phi_{2}\left(x_{1}, x_{2}, x_{3}\right)=\frac{1}{2}\left[x_{1}, x_{2}\right]+\frac{1}{2}\left[x_{1}, x_{3}\right]+\frac{1}{2}\left[x_{2}, x_{3}\right] \\
& \Phi_{3}\left(x_{1}, x_{2}, x_{3}\right)=\frac{1}{12}\left[x_{1},\left[x_{1}, x_{2}\right]\right]+\frac{1}{12}\left[x_{1},\left[x_{1}, x_{3}\right]\right]+\frac{1}{3}\left[x_{1},\left[x_{2}, x_{3}\right]\right]-\frac{1}{12}\left[x_{2},\left[x_{1}, x_{2}\right]\right] \\
&-\frac{1}{6}\left[x_{2},\left[x_{1}, x_{3}\right]\right]+\frac{1}{12}\left[x_{2},\left[x_{2}, x_{3}\right]\right]-\frac{1}{12}\left[x_{3},\left[x_{1}, x_{3}\right]\right]-\frac{1}{12}\left[x_{3},\left[x_{2}, x_{3}\right]\right],
\end{aligned}
$$

etc. This can be applied to get directly the so-called symmetric $\mathrm{BCH}$ formula,

$$
\exp \left(\frac{1}{2} X\right) \exp (Y) \exp \left(\frac{1}{2} X\right)=\exp (\Psi(X, Y))=\sum_{m=1}^{\infty} \Psi_{m}(X, Y)
$$


of great relevance in the design of time-symmetric splitting and composition methods (see e.g. [3], [13] and references therein). In this case it is easy to show that in general $\Psi_{m}(X, Y)=0$ when $m$ is even.

Of course, to get compact expressions we have to apply the same procedure as before to the corresponding formulas (15) with the obvious replacements $x_{1} \rightarrow X / 2$, $x_{2} \rightarrow Y, x_{3} \rightarrow X / 2$. It is more advantageous, however, to start with a different expression for (16) involving less terms before applying the reduction procedure. This can be achieved by connecting $\Phi(X, Y)$ and $\exp (\Psi(X, Y))$ as follows:

$$
\mathrm{e}^{\Psi(X, Y)}=\mathrm{e}^{-\frac{X}{2}} \mathrm{e}^{X} \mathrm{e}^{Y} \mathrm{e}^{\frac{X}{2}}=\mathrm{e}^{-\frac{X}{2}} \mathrm{e}^{\Phi(X, Y)} \mathrm{e}^{\frac{X}{2}},
$$

so that

$$
\Psi(X, Y)=\mathrm{e}^{-\operatorname{ad}_{\frac{X}{2}}} \Phi(X, Y)=\sum_{k=0}^{\infty} \frac{(-1)^{k}}{2^{k} k !} \operatorname{ad}_{X}^{k} \Phi(X, Y)
$$

where

$$
\operatorname{ad}_{A} B=[A, B], \quad \operatorname{ad}_{A}^{j} B=\left[A, \operatorname{ad}_{A}^{j-1} B\right], \quad \operatorname{ad}_{A}^{0} B=B .
$$

If we use the compact expressions for $\Phi_{m}(X, Y)$ obtained in the previous section, then the number of terms in the corresponding $\Psi_{m}$ determined according with (17) diminishes considerably. For instance, when $m=9$ we get 52 terms instead of 121 . By applying the existing identities, this number is further reduced to 42 . For comparison, $\Psi_{9}$ in the Hall basis contains 56 terms [7]

The last line in Table 1 contains the number of terms of $\Psi_{m}(X, Y)$ up to $m=9$, whereas the explicit expressions can also be found at [1].

On the other hand, formula (14) for $\Phi_{m}(X, Y)$ can be in fact obtained when the Magnus expansion is used to construct the formal solution of the differential equation

$$
Y^{\prime}(t)=A(t) Y(t), \quad Y(0)=I,
$$

when $A(t)$ is defined as

$$
A(t)=\theta(t-1) X+(\theta(t)+\theta(t-1)) Y,
$$

$\theta(t)$ being the step function. As is well known, the solution of (18) can be written as

$$
Y(t, 0)=\exp \Omega(t, 0)
$$

where $\Omega$ is an infinite series

$$
\Omega(t, 0)=\sum_{m=1}^{\infty} \Omega_{m}(t, 0), \quad \text { with } \quad \Omega_{m}(0,0)=0,
$$

whose terms are increasingly complex expressions involving time-ordered integrals of nested commutators of $A$ evaluated at different times. An explicit expression for $\Omega_{m}(t, 0), m \geq 1$, in terms of iterated integrals of linear combinations of independent commutators has been obtained in [2], namely

$$
\begin{gathered}
\Omega_{m}(t, 0)=\frac{1}{m} \sum_{\sigma \in S_{m-1}}(-1)^{d_{b}} \frac{1}{\left(\begin{array}{c}
m-1 \\
d_{b}
\end{array}\right)} \int_{0}^{t} d t_{1} \int_{0}^{t_{1}} d t_{2} \cdots \int_{0}^{t_{m-1}} d t_{m} \\
{\left[A\left(t_{\sigma(1)}\right),\left[A\left(t_{\sigma(2)}\right) \cdots\left[A\left(t_{\sigma(m-1)}\right), A\left(t_{m}\right)\right] \cdots\right]\right]}
\end{gathered}
$$

where $\sigma$ and $d_{\sigma}$ have the same meaning as in (13). Notice that, since $Y(t=2,0)=$ $\mathrm{e}^{X} \mathrm{e}^{Y}$, then $\log \left(\mathrm{e}^{X} \mathrm{e}^{Y}\right)=\Omega(2,0)$, and $\Omega_{m}(2,0)$ as given by (20) reproduces exactly the expression of $\Phi_{m}(X, Y)$ given by (14). This can be checked order by order. 


\section{Acknowledgements}

FC would like to thank the Isaac Newton Institute for Mathematical Sciences for support and hospitality during the programme "Geometry, compatibility and structure preservation in computational differential equations", when work on this paper was undertaken. This work was supported by EPSRC Grant Number EP/R014604/1 and by Ministerio de Economía y Competitividad (Spain) through project MTM201677660-P (AEI/FEDER, UE). We are also very grateful to Prof. M. Müger for bringing reference [10] to our attention and for providing us his lecture notes [21].

\section{A Appendix}

The following code in Mathematica implements (perhaps not in the most efficient way) formula (7):

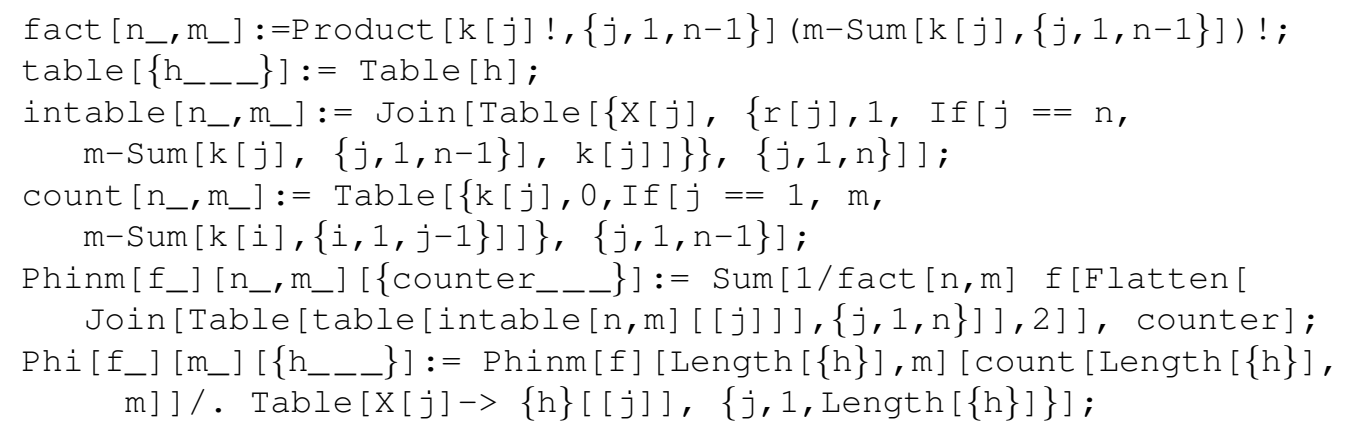

Next we compute $\Phi_{m}\left(X_{1}, \ldots, X_{n}\right)$ with the explicit expression (9) for $\varphi_{m}$ in terms of non-commutative products Mm. This is contained in PhiMm [m] $\left[\left\{X_{1}, \ldots, X_{n}\right\}\right]$ :

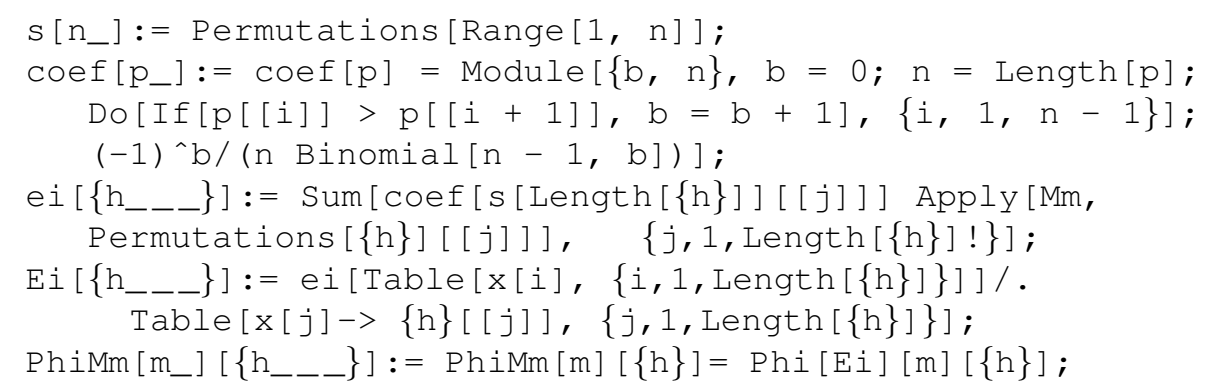

Then we express $\varphi_{n}\left(X_{1}, \ldots, X_{n}\right)$ in terms of commutators, equation (13), and finally $\Phi_{m}$ by computing PhiCmt $[\mathrm{m}]\left[\left\{X_{1}, \ldots, X_{n}\right\}\right]$ :

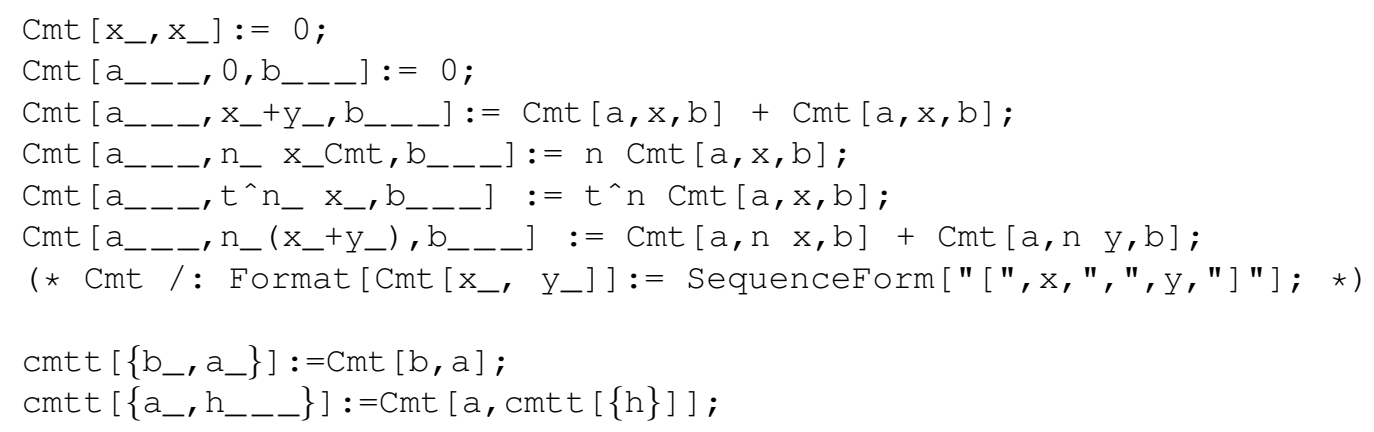




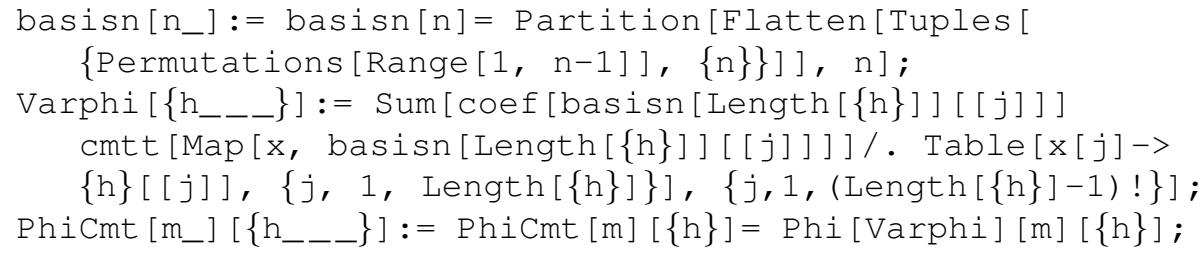

The first block defines the commutator (just the linearity property and the antisymmetry) with the correct format for output if necessary. Linearity properties in an analogous way should be implemented for the non commutative product $\mathrm{Mm}$. The second block defines the basis and the generic term $\varphi_{n}\left(X_{1}, \ldots, X_{n}\right)$. Finally, let us remark that since the number of variables is free, the same code allows one to compute both the $\mathrm{BCH}$ PhiCmt [m] [ $\{X, Y\}]$ and the symmetric BCH series, PhiCmt [m] [ $\left.\left\{\frac{1}{2} X, Y, \frac{1}{2} X\right\}\right]$.

\section{B Appendix}

The algorithm we have applied to generate the identities among commutators and a basis of the homogeneous subspace $\mathcal{L}_{m}(X, Y)$ formed by right-nested commutators is a generalization of a procedure proposed in [17], and can be summarized as follows:

For each $j=2, \ldots, m$, do

1. Generate all possible right-nested commutators $C_{i}$ involving $j$ operators $X$ and $m-j$ operators $Y$. For example, with $m=4$,

$$
\mathcal{B}_{4}=\{[X,[X,[X, Y]]],[Y,[X,[X, Y]]],[X,[Y,[X, Y]]],[Y,[Y,[X, Y]]]\}
$$

2. Generate the corresponding element $\left\langle C_{i}\right\rangle$ in the homogeneous subspace $\mathcal{U}_{m}(X, Y)$ of the universal enveloping algebra associated with $\mathcal{L}(X, Y)$. This is done by expanding each commutator $[A, B]=A B-B A$. For example, on the previous list, for $C_{1}=[X,[X,[X, Y]]]$,

$$
<C_{1}>=X X X Y-3 X X Y X+3 X Y X X-Y X X X
$$

3. The element $\left\langle C_{i}\right\rangle$ is then a linear combination of words $X_{i_{1}} X_{i_{2}} \cdots X_{i_{m}}$, where $X_{i_{j}}$ is either $X$ of $Y$. The total number of words is $\left(\begin{array}{c}m \\ j\end{array}\right)$. Once all these words are arranged in a prescribed order, the element commutator $C_{i}$ can be identified with the vector $\left(a_{1}, a_{2}, \ldots, a_{p}\right)$ formed by the linear combination. Then $C_{1}$ would be $C_{1} \equiv(1,-3,0,3,0,0,-1,0,0,0,0,0)$.

4. Define a matrix $A$ whose rows are formed by these coefficient vectors and augment it to the right with the $m \times m$ identity matrix, forming an block matrix $(A \mid I)$. Apply Gauss-Jordan elimination and get the block matrix $(M \mid P)$. For $m=4$ we have,

$$
(A \mid I)=\left(\begin{array}{cccccccccccc|cccc}
1 & -3 & 0 & 3 & 0 & 0 & -1 & 0 & 0 & 0 & 0 & 0 & 1 & 0 & 0 & 0 \\
0 & 0 & -1 & 0 & 2 & 0 & 0 & -2 & 0 & 1 & 0 & 0 & 0 & 1 & 0 & 0 \\
0 & 0 & -1 & 0 & 2 & 0 & 0 & -2 & 0 & 1 & 0 & 0 & 0 & 0 & 1 & 0 \\
0 & 0 & 0 & 0 & 0 & 1 & 0 & 0 & -3 & 0 & 3 & -1 & 0 & 0 & 0 & 1
\end{array}\right)
$$


and

$$
(M \mid P)=\left(\begin{array}{cccccccccccc|cccc}
1 & -3 & 0 & 3 & 0 & 0 & -1 & 0 & 0 & 0 & 0 & 0 & 1 & 0 & 0 & 0 \\
0 & 0 & 1 & 0 & -2 & 0 & 0 & 2 & 0 & -1 & 0 & 0 & 0 & 0 & -1 & 0 \\
0 & 0 & 0 & 0 & 0 & 1 & 0 & 0 & -3 & 0 & 3 & -1 & 0 & 0 & 0 & 1 \\
0 & 0 & 0 & 0 & 0 & 0 & 0 & 0 & 0 & 0 & 0 & 0 & 0 & 1 & -1 & 0
\end{array}\right)
$$

5. Now the non-vanishing rows on $M$ give the commutators of the basis. The identities we want to find out are obtained after making equal to zero the linear combinations on $P \cdot \mathcal{B}_{n}$ corresponding to the vanishing rows. In our example, since there is one null row on $M$ we get one Grade 4 identity, we equal to zero the last element on the product $P \cdot \mathcal{B}_{4}$, that is,

$$
0=[Y,[X,[X, Y]]]-[X,[Y,[X, Y]]] .
$$

\section{Appendix}

For completeness, we include in the sequel the explicit compact expression we have obtained for $\Phi_{m}(X, Y), m=1, \ldots, 10$. Here $\left[X^{p_{1}} Y^{q_{1}} \ldots X^{p_{m}} Y^{q_{m}}\right]$ denotes the right-nested commutator based on the word $X^{p_{1}} Y^{q_{1}} \ldots X^{p_{m}} Y^{q_{m}}$. Thus, in particular,

$$
\left[Y^{2} X^{4} Y\right] \equiv[Y,[Y,[X,[X,[X,[X, Y]]]]]] .
$$

$$
\begin{aligned}
\Phi_{1}(X, Y) & =X+Y \\
\Phi_{2}(X, Y) & =\frac{1}{2}[X Y] \\
\Phi_{3}(X, Y) & =\frac{1}{12}\left[X^{2} Y\right]-\frac{1}{12}[Y X Y] \\
\Phi_{4}(X, Y) & =-\frac{1}{24}[X Y X Y] \\
\Phi_{5}(X, Y) & =-\frac{1}{720}\left[X^{4} Y\right]-\frac{1}{120}\left[X Y X^{2} Y\right]-\frac{1}{360}\left[X Y^{2} X Y\right]+\frac{1}{360}\left[Y X^{3} Y\right] \\
& +\frac{1}{120}\left[Y^{2} X^{2} Y\right]+\frac{1}{720}\left[Y^{3} X Y\right] \\
\Phi_{6}(X, Y) & =-\frac{1}{720}\left[X^{2} Y^{2} X Y\right]+\frac{1}{240}\left[X Y^{2} X^{2} Y\right]+\frac{1}{1440}\left[X Y^{3} X Y\right]+\frac{1}{1440}\left[Y X^{4} Y\right] \\
\Phi_{7}(X, Y) & =\frac{1}{30240}\left[X^{6} Y\right]+\frac{1}{5040}\left[X^{2} Y X^{3} Y\right]-\frac{1}{10080}\left[X^{2} Y^{2} X^{2} Y\right] \\
& +\frac{1}{10080}\left[X Y X^{4} Y\right]+\frac{1}{1008}\left[X Y X Y X^{2} Y\right]+\frac{1}{5040}\left[X Y X Y^{2} X Y\right] \\
& -\frac{1}{7560}\left[X Y^{2} X^{3} Y\right]+\frac{1}{3360}\left[X Y^{3} X^{2} Y\right]+\frac{1}{10080}\left[X Y^{4} X Y\right] \\
& -\frac{1}{10080}\left[Y X^{5} Y\right]-\frac{1}{1260}\left[Y X Y X^{3} Y\right]-\frac{1}{1680}\left[Y X Y^{2} X^{2} Y\right] \\
& +\frac{1}{3360}\left[Y^{2} X^{4} Y\right]-\frac{1}{3360}\left[Y^{2} X Y X^{2} Y\right]-\frac{1}{2520}\left[Y^{2} X Y^{2} X Y\right] \\
& +\frac{1}{7560}\left[Y^{3} X^{3} Y\right]+\frac{1}{10080}\left[Y^{4} X^{2} Y\right]-\frac{1}{30240}\left[Y^{5} X Y\right]
\end{aligned}
$$




$$
\begin{aligned}
& \left.\Phi_{8}(X, Y)=-\frac{5}{24192}\left[X^{3} Y^{3} X Y\right]+\frac{1}{2520}\left[X^{2} Y X Y^{2} X Y\right]\right]+\frac{1}{20160}\left[X^{2} Y^{4} X Y\right] \\
& +\frac{1}{15120}\left[X Y X^{2} Y^{2} X Y\right]-\frac{1}{2016}\left[X Y X Y^{2} X^{2} Y\right]-\frac{1}{20160}\left[X Y X Y^{3} X Y\right] \\
& +\frac{1}{20160}\left[X Y^{2} X Y X^{2} Y\right]-\frac{1}{10080}\left[X Y^{2} X Y^{2} X Y\right]-\frac{1}{60480}\left[X Y^{5} X Y\right] \\
& -\frac{1}{60480}\left[Y X^{6} Y\right]+\frac{1}{20160}\left[Y X^{3} Y X^{2} Y\right]-\frac{1}{5040}\left[Y X^{2} Y X^{3} Y\right]+\frac{1}{20160}\left[Y^{2} X^{5} Y\right] \\
& \Phi_{9}(X, Y)=-\frac{1}{302400}\left[X^{5} Y X^{2} Y\right]+\frac{1}{113400}\left[X^{5} Y^{2} X, Y\right]-\frac{1}{40320}\left[X^{4} Y^{2} X^{2} Y\right] \\
& -\frac{1}{120960}\left[X^{4} Y^{3} X Y\right]+\frac{13}{604800}\left[X^{3} Y^{4} X Y\right]+\frac{1}{24192}\left[X^{2} Y X^{2} Y X^{2} Y\right] \\
& +\frac{1}{30240}\left[X^{2} Y X^{2} Y^{2} X Y\right]-\frac{1}{60480}\left[X^{2} Y X Y X^{3} Y\right]-\frac{1}{20160}\left[X^{2} Y X Y^{3} X Y\right] \\
& -\frac{11}{604800}\left[X^{2} Y^{5} X Y\right]-\frac{1}{151200}\left[X Y X^{6} Y\right]-\frac{1}{20160}\left[X Y X^{3} Y X^{2} Y\right] \\
& -\frac{1}{10080}\left[X Y X^{2} Y^{2} X^{2} Y\right]+\frac{1}{40320}\left[X Y X^{2} Y^{3} X Y\right]+\frac{1}{18900}\left[X Y X Y^{4} X Y\right] \\
& -\frac{1}{20160}\left[X Y^{2} X Y^{2} X^{2} Y\right]-\frac{1}{17280}\left[X Y^{2} X Y^{3} X Y\right]-\frac{1}{120960}\left[X Y^{4} X^{3} Y\right] \\
& -\frac{1}{302400}\left[X Y^{6} X Y\right]+\frac{1}{302400}\left[Y X^{7} Y\right]+\frac{1}{50400}\left[Y X^{4} Y X^{2} Y\right] \\
& +\frac{1}{120960}\left[Y X^{4} Y^{2} X Y\right]+\frac{1}{20160}\left[Y X^{2} Y X Y X^{2} Y\right]-\frac{1}{40320}\left[Y X Y^{2} X^{4} Y\right] \\
& +\frac{1}{10080}\left[Y X Y^{2} X Y X^{2} Y\right]+\frac{1}{30240}\left[Y X Y^{2} X Y^{2} X Y\right]+\frac{1}{151200}\left[Y X Y^{5} X Y\right] \\
& +\frac{1}{302400}\left[Y^{2} X^{6} Y\right]+\frac{1}{20160}\left[Y^{2} X Y X^{4} Y\right]-\frac{1}{30240}\left[Y^{2} X Y^{2} X^{3} Y\right] \\
& +\frac{1}{60480}\left[Y^{2} X Y^{3} X^{2} Y\right]-\frac{13}{604800}\left[Y^{3} X^{5} Y\right]-\frac{1}{90720}\left[Y^{3} X^{2} Y^{2} X Y\right] \\
& +\frac{1}{120960}\left[Y^{4} X^{4} Y\right]+\frac{1}{453600}\left[Y^{5} X^{3} Y\right]+\frac{1}{302400}\left[Y^{6} X^{2} Y\right] \\
& +\frac{1}{1209600}\left[Y^{7} X Y\right]-\frac{1}{1209600}\left[X^{8} Y\right]
\end{aligned}
$$




$$
\begin{aligned}
& \Phi_{10}(X, Y)=-\frac{1}{2419200}\left[X^{4} Y X^{4} Y\right]-\frac{1}{604800}\left[X^{4} Y^{2} X^{3} Y\right]+\frac{1}{604800}\left[X^{3} Y X^{5} Y\right] \\
& +\frac{1}{151200}\left[X^{3} Y X Y X^{3} Y\right]+\frac{1}{604800}\left[X^{3} Y^{2} X^{4} Y\right]+\frac{1}{134400}\left[X^{3} Y^{2} X Y X^{2} Y\right] \\
& +\frac{1}{362880}\left[X^{3} Y^{3} X^{3} Y\right]+\frac{1}{134400}\left[X^{3} Y^{4} X^{2} Y\right]-\frac{1}{403200}\left[X^{2} Y X^{6} Y\right] \\
& -\frac{1}{100800}\left[X^{2} Y X^{2} Y X^{3} Y\right]-\frac{1}{201600}\left[X^{2} Y X Y X^{4} Y\right]-\frac{1}{50400}\left[X^{2} Y X Y X Y X^{2} Y\right] \\
& -\frac{1}{604800}\left[X^{2} Y X Y X Y^{2} X Y\right]-\frac{1}{302400}\left[X^{2} Y X Y^{2} X^{3} Y\right]-\frac{1}{80640}\left[X^{2} Y X Y^{3} X^{2} Y\right] \\
& -\frac{1}{100800}\left[X^{2} Y^{2} X Y X^{3} Y\right]-\frac{1}{201600}\left[X^{2} Y^{2} X Y^{2} X^{2} Y\right]+\frac{1}{403200}\left[X^{2} Y^{3} X^{4} Y\right] \\
& -\frac{1}{75600}\left[X^{2} Y^{3} X Y X^{2} Y\right]-\frac{1}{362880}\left[X^{2} Y^{3} X Y^{2} X Y\right]+\frac{1}{403200}\left[X^{2} Y^{4} X^{3} Y\right] \\
& -\frac{1}{134400}\left[X^{2} Y^{5} X^{2} Y\right]-\frac{1}{604800}\left[X^{2} Y^{6} X Y\right]+\frac{1}{604800}\left[X Y X^{7} Y\right] \\
& +\frac{1}{40320}\left[X Y X^{2} Y X^{4} Y\right]+\frac{1}{67200}\left[X Y X^{2} Y X Y X^{2} Y\right]-\frac{1}{151200}\left[X Y X^{2} Y^{2} X^{3} Y\right] \\
& -\frac{1}{50400}\left[X Y X Y X^{5} Y\right]+\frac{1}{25200}\left[X Y X Y X Y X^{3} Y\right]+\frac{1}{25200}\left[X Y X Y X Y^{2} X^{2} Y\right] \\
& -\frac{1}{100800}\left[X Y X Y^{2} X^{4} Y\right]-\frac{1}{100800}\left[X Y X Y^{2} X Y X^{2} Y\right]+\frac{1}{302400}\left[X Y X Y^{2} X Y^{2} X Y\right] \\
& +\frac{1}{75600}\left[X Y X Y^{3} X^{3} Y\right]+\frac{1}{50400}\left[X Y X Y^{4} X^{2} Y\right]+\frac{1}{151200}\left[X Y^{2} X^{6} Y\right] \\
& -\frac{1}{100800}\left[X Y^{2} X^{2} Y X^{3} Y\right]-\frac{1}{50400}\left[X Y^{2} X^{2} Y^{2} X^{2} Y\right]+\frac{1}{403200}\left[X Y^{2} X Y X^{4} Y\right] \\
& +\frac{1}{25200}\left[X Y^{2} X Y X Y X^{2} Y\right]+\frac{1}{151200}\left[X Y^{2} X Y X Y^{2} X Y\right]-\frac{1}{43200}\left[X Y^{2} X Y^{2} X^{3} Y\right] \\
& -\frac{1}{67200}\left[X Y^{2} X Y^{3} X^{2} Y\right]+\frac{1}{100800}\left[X Y^{2} X Y^{4} X Y\right]-\frac{1}{75600}\left[X Y^{3} X Y X^{3} Y\right] \\
& -\frac{1}{302400}\left[X Y^{3} X Y^{2} X^{2} Y\right]+\frac{13}{1209600}\left[X Y^{4} X^{4} Y\right]+\frac{1}{75600}\left[X Y^{4} X Y X^{2} Y\right] \\
& -\frac{1}{60480}\left[X Y^{4} X Y^{2} X Y\right]-\frac{1}{226800}\left[X Y^{5} X^{3} Y\right]+\frac{1}{86400}\left[X Y^{6} X^{2} Y\right]+\frac{1}{2419200}\left[X Y^{7} X Y\right]
\end{aligned}
$$

\section{References}

[1] http://www.gicas.uji.es/Research/bch.html.

[2] A. Arnal, F. Casas, And C. Chiralt, A general formula for the Magnus expansion in terms of iterated integrals of right-nested commutators, J. Phys. Commun., 2 (2018), p. 035024.

[3] S. Blanes And F. CASAs, A Concise Introduction to Geometric Numerical Integration, CRC Press, 2016. 
[4] A. Bonfiglioli, An ODE's version of the formula of Baker, Campbell, Dynkin and Hausdorff and the construction of Lie groups with prescribed Lie algebra, Mediterr. J. Math., 7 (2010), pp. 387-414.

[5] A. Bonfiglioli And R. Fulci, Topics in Noncommutative Algebra. The Theorem of Campbell, Baker, Hausdorff and Dynkin, vol. 2034 of Lecture Notes in Mathematics, Springer, 2012.

[6] E. Burgunder, Eulerian idempotent and Kashiwara-Vergne conjecture, Ann. Inst. Fourier, 58 (2008), pp. 1153-1184.

[7] F. CASAS AND A. Murua, An efficient algorithm for computing the BakerCampbell-Hausdorff series and some of its applications, J. Math. Phys., 50 (2009), p. 033513.

[8] A. Dragt And E. Forest, Computation of nonlinear behavior of Hamiltonian systems using Lie algebraic methods, J. Math. Phys., 24 (1983), pp. 2734-2744.

[9] E. Dynkin, Evaluation of the coefficients of the Campbell-Hausdorff formula, Dokl. Akad. Nauk. SSSR, 57 (1947), pp. 323-326.

[10] E. Dynkin, On the representation by means of commutators of the series $\log \left(e^{x} e^{y}\right)$ for noncommutative $x$ and $y$, Mat. Sb. (N.S.), 25(67) (1949), pp. 155162 (in Russian).

[11] E. Dynkin, Calculation of the coefficients in the Campbell-Hausdorff series, in Selected Papers of E.B. Dynkin with Commentary, E. Dynkin, A. Yushkevich, G. Seitz, and A. Onishchik, eds., American Mathematical Society, 2000, pp. 3135 .

[12] K. Goldberg, The formal power series for $\log \left(\mathrm{e}^{x} \mathrm{e}^{y}\right)$, Duke Math. J., 23 (1956), pp. 13-21.

[13] E. Hairer, C. Lubich, AND G. WANnER, Geometric Numerical Integration. Structure-Preserving Algorithms for Ordinary Differential Equations, SpringerVerlag, Second ed., 2006.

[14] J. Helmstetter, Série de Hausdorff, d'une algèbre de Lie et projections canoniques de l'algèbre enveloppante, J. Algebra, 120 (1989), pp. 170-199.

[15] H. Kobayashi, N. Hatano, And M. Suzuki, Goldberg's theorem and the Baker-Campbell-Hausdorff formula, Physica A, 250 (1998), pp. 535-548.

[16] M. Kolsrud, Maximal reductions in the Baker-Hausdorff formula, J. Math. Phys., 34 (1993), pp. 270-285.

[17] R.-C. LI, Raising the order of unconventional schemes for ordinary differential equations, $\mathrm{PhD}$ thesis, University of California at Berkeley, 1995.

[18] J.-L. LODAY, Opérations sur l'homologie cyclique des algèbres commutatives, Invent. Math., 96 (1989), pp. 205-230. 
[19] J.-L. LodAy, Série de Hausdorff, idempotents Eulériens et algèbres de Hopf, Expo. Math., 12 (1994), pp. 165-178.

[20] R. McLachlan And A. Murua, The Lie algebra of classical mechanics, tech. rep., arXiv:1905.07554, 2019.

[21] M. MÜGER, Notes on the theorem of Baker-Campbell-Hausdorff-Dynkin, https://www.math.ru.nl/ mueger/PDF/BCHD.pdf.

[22] J. OTEO, The Baker-Campbell-Hausdorff formula and nested commutator identities, J. Math. Phys., 32 (1991), pp. 419-424.

[23] C. Reutenauer, Free Lie Algebras, vol. 7, Oxford University Press, 1993.

[24] L. Solomon, On the Poincaré-Birkhoff-Witt theorem, J. Comb. Theory, 4 (1968), pp. 363-375.

[25] A. SORnborger AND E. STEWART, Higher-order methods for simulations on quantum computers, Phys. Rev. A, 60 (1999), pp. 1956-1965.

[26] R. S. STRICHARTZ, The Campbell-Baker-Hausdorff-Dynkin formula and solutions of differential equations, J. Funct. Anal., 72 (1987), pp. 320-345.

[27] V. Varadarajan, Lie Groups, Lie Algebras, and Their Representations, Springer-Verlag, 1984.

[28] R. WiLCOX, Exponential operators and parameter differentiation in quantum physics, J. Math. Phys., 8 (1967), pp. 962-982. 